\title{
Ultrafast pulse generation in photoconductive switches
}

\section{Keil, Ulrich Dieter Felix; Dykaar, D. R.}

Published in:

I E E E Journal of Quantum Electronics

Link to article, DOI:

10.1109/3.535372

Publication date:

1996

Document Version

Publisher's PDF, also known as Version of record

Link back to DTU Orbit

Citation (APA):

Keil, U. D. F., \& Dykaar, D. R. (1996). Ultrafast pulse generation in photoconductive switches. I E E E Journal of Quantum Electronics, 32, 1664-1671. https://doi.org/10.1109/3.535372

\section{General rights}

Copyright and moral rights for the publications made accessible in the public portal are retained by the authors and/or other copyright owners and it is a condition of accessing publications that users recognise and abide by the legal requirements associated with these rights.

- Users may download and print one copy of any publication from the public portal for the purpose of private study or research.

- You may not further distribute the material or use it for any profit-making activity or commercial gain

- You may freely distribute the URL identifying the publication in the public portal

If you believe that this document breaches copyright please contact us providing details, and we will remove access to the work immediately and investigate your claim 


\title{
Ultrafast Pulse Generation in Photoconductive Switches
}

\author{
U. D. Keil and D. R. Dykaar, Senior Member, IEEE
}

\begin{abstract}
Carrier and field dynamics in photoconductive switches are investigated by electrooptic sampling and voltagedependent reflectivity measurements. We show that the nonuniform field distribution due to the two-dimensional nature of coplanar photoconductive switches, in combination with the large difference in the mobilities of holes and electrons, determine the pronounced polarity dependence. Our measurements indicate that the pulse generation mechanism is a rapid voltage breakdown across the photoconductive switch and not a local field breakdown.
\end{abstract}

\section{INTRODUCTION}

$\mathbf{T}$ HE common excitation geometry for a photoconductive switch (PCS) is the illumination of the whole gap with the short laser pulse. The photogenerated charge carriers reduce the resistivity of the substrate material and the voltage across the gap drops. The measured rise time is determined by the laser measurement system and the fall time determined by the carrier lifetime. Photoconductive switches on material with short carrier lifetimes have been shown to generate electrical pulses as short as $600 \mathrm{fs}$ [1] when excited with 100 -fs optical pulses. The substrate material used in these experiments is low-temperature grown (LT) GaAs, i.e., a material with a short carrier lifetime. This material, however, complicates the production process of active devices on the same wafer. An alternative approach for generating short electrical pulses is asymmetric excitation of photoconductive switches on long carrier lifetime materials. The first observation was a $350-\mathrm{fs}$ electrical pulse generated on Silicon on Sapphire (SOS) [2]. Pulse widths as short as $200 \mathrm{fs}$ on LT GaAs and 250 fs on semi-insulating (SI) GaAs have been measured by avoiding broadening through propagation effects [3]. All the above results were obtained with electrooptic (e/o) sampling. It was found that pulse amplitudes at the positive electrode were significantly larger than at the negative electrode [2], [4]. The most detailed experimental analysis of position dependence has been presented in [4]

The literature contains several attempts to clarify the generation mechanism and the special role of the anode. Generation of the short pulse has been explained as a local field screening by photogenerated carriers while the voltage was assumed to be constant (in time) across the gap [5]. The constant voltage requires a field increase at nonilluminated regions.

Manuscript received July 7, 1995; revised April 30, 1996.

U. D. Keil is with Mikroelektronik Centret DTU, DK 2800 Lyngby, Denmark.

D. R. Dykaar is with Bell Laboratories, Lucent Technologies, Murray Hill, NJ, 07974-2070 USA.

Publisher Item Identifier S 0018-9197(96)06287-2.
As the carriers spread, certain regions exist where the field first decreases and then increases rapidly. An extension of this model involving Monte Carlo simulations reports current transients of about $1 \mathrm{ps}$ [6]; this is $(5 \times)$ too long to explain the observed 200-fs pulses. These models treat the problem in one dimension and thus ignore the field enhancement at the electrodes due to the two-dimensional (2-D) geometry of a coplanar PCS. In addition, it is assumed that the voltage across the PCS stays constant after the injection of photocarriers. An explanation for the asymmetry is not provided by these theories, unless a sheet of carriers at the electrodes is introduced artificially [6]. Before recent measurements showed that the enhancement of the short pulse generation at the anode is observed on a variety of semiconductors, a material specific explanation had been considered for SOS [2]. A ptype autodoping was assumed which would indeed result in a higher field at the Schottky contact of the anode.

A similar asymmetry was observed in pulsed terahertz radiation [7], [8]. The terahertz measurements were reported for transmission line structures of $50-100 \mu \mathrm{m}$ spacing of the electrodes. The authors attributed the asymmetry to a trapenhanced field at the positive electrode for SI GaAs. As much as this field enhancement convincingly explains the asymmetry in those measurements, it only applies to SI GaAs specifically. Furthermore, the authors report that for an $80-\mu \mathrm{m}$ gap $90 \%$ of the applied potential is within $5 \mu \mathrm{m}$ from the anode; this is the typical electrode spacing here. Our measurements are sensitive to both the static and the transient field. We directly measure the static field and characterize the asymmetry for small $(5-\mu \mathrm{m})$ electrode spacings as well as for a variety of semiconductor substrates.

From an application standpoint, it is important to characterize photoconductive switches integrated in coplanar strip lines with small dimensions. Coplanar strip lines with $5-\mu \mathrm{m}$ width and spacing represent the best compromise in terms of low attenuation and minimal dispersion for bandwidths up to 2 THz. Radiation losses are small due to the small spacing and metal losses are small due to the relatively wide conductor [9].

In this paper, we present experimental results that resolve, or at least rule out, some of the various effects reported to contribute to the short-pulse generation under asymmetric excitation. The paper is structured in the following way. After the experimental details, we present the system-limited response generated by asymmetric excitation and measured with electrooptic sampling. We measure the dependencies on the power and on the electric field to test for optical nonlinearity effects followed by measurements of the influence of the pump 


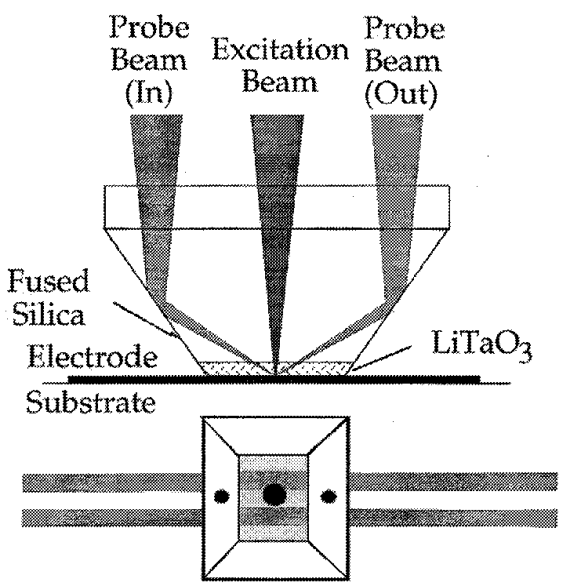

Fig. 1. Side and top view of the TIR electrooptic sampling probe. The view from the top is shown for overlapping pump and probe beams.

wavelength. The crucial parts are the investigation of the polarity, pump position, and probe-position dependencies. All of these measurements were done with electrooptic sampling. In the final experiment, we investigate the position-dependent change in reflectivity without the invasive electrooptic crystal.

\section{EXPERIMENTAL}

The investigated samples consist of Au coplanar strip lines with $5-\mu \mathrm{m}$ (or $10-\mu \mathrm{m}$ where stated) contact width and spacing evaporated on semiconductor substrates to form a photoconductive switch. The substrates are InP and SI GaAs with and without MBE grown LT GaAs, n- and p-doped GaAs layers.

Most of the measurements reported here use e/o sampling in a total internal reflection (TIR) geometry (Fig. 1) [3]. The TIR geometry allows measurement of the electrical transient at the excitation site, i.e., at zero propagation distances. The use of a single microscope objective $(20 \times)$ for pump and probe beam allows high spatial resolution with spot sizes as small as $2 \mu \mathrm{m}$. The limit of magnification is set by the microscope objective. The field of view has to include the reflecting facets for the incoming and outgoing probe beam. Another constraint on the spatial resolution is that the probe beam integrates field changes, not only at the spot where it reflects from the bottom facet, but along the whole path through the e/o crystal. Due to the physical extent of the sampling crystal, fringing fields are also measured which limits the spatial resolution (and hence the temporal resolution). For the measurement with the highest temporal resolution, the e/o-crystal was polished down to 37 $\mu \mathrm{m}$; this thickness is still large compared to the size of the PCS gap of $5 \mu \mathrm{m}$. The bottom facets are $100 \mu \mathrm{m} \times 100 \mu \mathrm{m}$. For measurements with a longer time window (but correspondingly less time resolution), a TIR probe with $100-\mu$ m thickness, $300-\mu \mathrm{m}$ width, and $200-\mu \mathrm{m}$ length is used. The larger inplane dimension allows a longer reflection-free time window. Reflections from the impedance mismatch at the edges of the sampling crystal are thus delayed.

In e/o sampling, the optical axis of the sampling crystal is conventionally chosen to be sensitive to the lateral electric field, i.e., the field perpendicular to the transmission lines.
Therefore, we do not detect the enhancement of the field magnitude at the electrodes, which is due to perpendicular field components.

\section{EleCtrooptic SAMPLing RESUlts}

Electrooptic sampling with zero propagation distance and asymmetric excitation can resolve electrical pulse widths down to $200 \mathrm{fs}$ [3]. The results in Fig. 2 are measured with a prism-compensated colliding-pulse mode-locked (CPM) laser operating typically with 80 -fs pulses and a wavelength of $620 \mathrm{~nm}$. In order to achieve the highest possible temporal resolution, the $37-\mu \mathrm{m}$ thick e/o probe is used together with an extra-cavity prism pair to compensate for the dispersion of the optical elements. To further minimize dispersion of the laser beam by external optical components, we avoid using acoustooptic modulators for modulating the pump beam but instead modulate the applied voltage, $V_{0}$. The autocorrelation with a $\mathrm{LiIO}_{3}$ crystal at the sample position, including dispersing effects of all optics in the beam path, is measured to be $170 \mathrm{fs}$. This limit and the symmetric shape of the pulses in the e/o signal (same rise and fall time in Fig. 2) indicate that the measured pulse widths are limited by the resolution of the system. All measurements are made in a gapless [10] (sliding contact [11]) switch configuration. With the voltage modulated between 0 and $V_{0}$, the raw data include an offset representing the associated field change. If the fields for zero bias (surface and Schottky depletion fields) are negligible, then the signal is the field for bias $V_{0}$. (This also implies that all time constants of the photogenerated effects are shorter than $12 \mathrm{~ns}$, the repetition period of the laser.) For uniform illumination, the photogenerated carriers reduce the gap resistance and cause a transient drop in the voltage across the PCS. For clarity, unless otherwise noted, we show this transient part as a positive signal and subtract the $V_{0}$ component.

The measured pulse waveforms strongly depend on the position of the beams. The electrical transients shown in Fig. 2 are measured with the pump beam slightly overlapping the positive electrode and the probe beam placed as close as possible to the pump beam, thus avoiding any propagation effects. The signal at $1.8 \mathrm{ps}$ arises from reflections in the $\mathrm{LiTaO}_{3}$ crystal [12].

Fig. 2(a) and (b) compare the results for LT GaAs [9] and SI GaAs. In both cases, we measure a resolution-limited response. In the second measurement, the CPM laser was not quite optimized to generate the shortest possible pulses. In particular, the comparison shows that the electrical transient in the first picosecond does not depend on the charge carrier lifetime of the substrate but is determined by the excitation scheme.

\section{PUMP-POWER DEPENDENCE}

The observed e/o signal in Fig. 2 is instantaneous with respect to the available time resolution as would be expected for a nonlinear optical effect. One possibility is, therefore, that a field generated by optical rectification couples into the transmission line or is directly detected in the $\mathrm{LiTaO}_{3}$ crystal. We performed measurements at different pump power levels to 


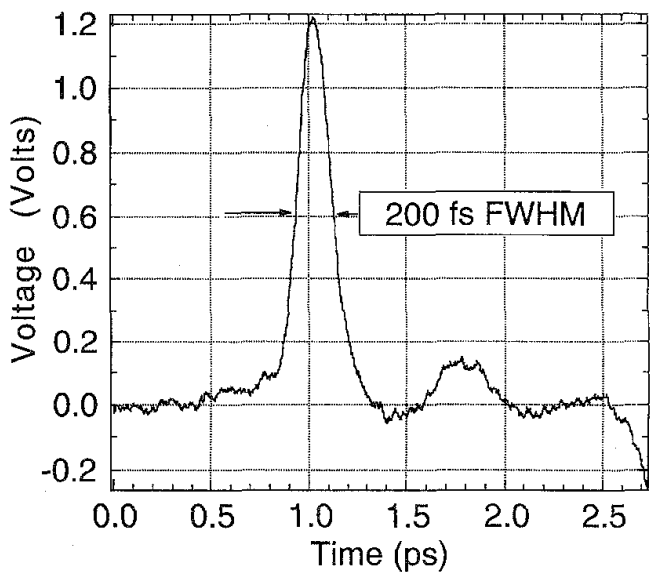

(a)

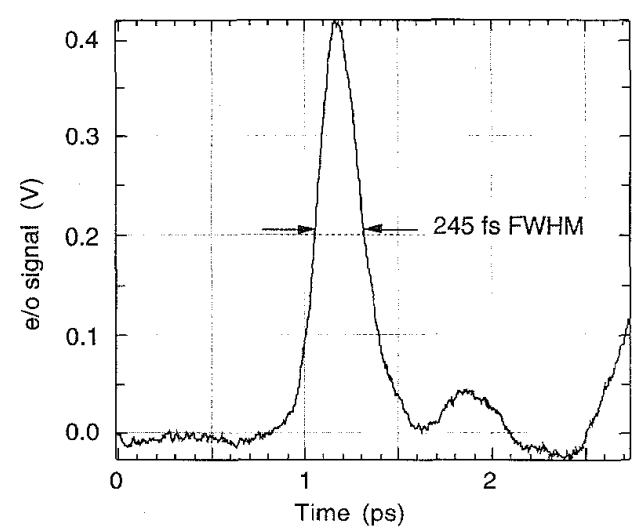

(b)

Fig. 2. Electrooptically sampled data for (a) LT GaAs (pulse width is 200 -fs FWHM and rise time is $150 \mathrm{fs}$ ), and (b) GaAs (245-fs FWHM pulse width).

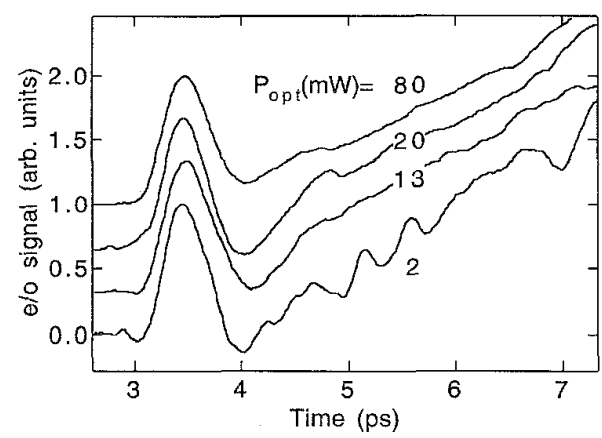

Fig. 3. Dependence on pump power. Amplitudes are normalized to the peak at $3.5 \mathrm{ps}$, and different offsets are added to the curves. The measurement is taken with a $\mathrm{Ti}$ : sapphire laser at $760 \mathrm{~nm}, V_{0}=10 \mathrm{~V}$.

resolve effects that depend nonlinearly on the optical power. The dependencies are demonstrated in Figs. 3 and 4. These measurements, and all the remaining ones, were taken with a $100-\mu \mathrm{m}$-thick, $200-\mu \mathrm{m}$-long, and $300-\mu \mathrm{m}$-wide e/o crystal. The use of this crystal limits the temporal resolution, so the minimum pulse widths are now about $500 \mathrm{fs}$, but allows measurement of a longer time range as reflections from the crystal boundaries are delayed.

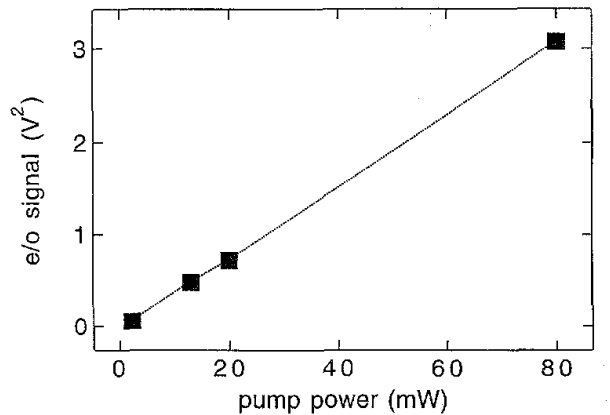

Fig. 4. Amplitude dependence of the e/o-signals in Fig. 3.

Fig. 3 shows that the observed pulse shapes do not depend on pump power. The e/o signals in Fig. 3 are normalized to the peak at $3.5 \mathrm{ps}$ and show that there is no qualitative difference for different pump powers. Also, if the rise time of the slow component resembles a recharging of the PCS's capacitance, then this rise time should strongly depend on the injected carrier density [13]. Fig. 4 shows a plot of the squared amplitudes versus pump power. For the amplitudes of the pulse, an excellent fit can be obtained by assuming $V_{e o}^{2} \propto P_{o p t}$ or a linear dependence on the optical field: $V_{e o} \propto E_{\omega}$.

This would imply a "dc" or rectified signal is induced by the optical field. However, optical rectification (and any possible difference frequency mixing) requires at least two optical fields to obtain a difference of zero frequency, making this an unlikely explanation. In addition, such an effect would depend on the crystallographic orientation, as has been shown previously [17].

On the other hand, one can assume that the signal is due to photogenerated carriers. Neglecting saturation effects, the number of carriers, $N$, is proportional to $P_{\text {opt }}$ which in turn is measured to be proportional to the square of the generated signal, $V_{e o}^{2}$. However, based on these measurements, we can neither rule out saturation effects nor explain how the finite energy stored in the PCS (forming a capacitor as discussed in Section VII-B) affects the result. Furthermore, diffraction at the electrode edge causes a significant amount of light to be coupled under the electrode, where the field is smaller, as well as decreasing the amount of light immediately adjacent to the electrode. More work is required to sort out this aspect of the effect.

\section{DEPENDENCE ON APPLIED FIELD}

The linear dependence on the optical field can be an indication of an electrooptic effect. Therefore, we test the relation to the applied field. Fig. 5 shows e/o data measured with different applied voltages, $V_{0}$. The data are divided by $V_{0}$. In this way, all linear dependencies on $V_{0}$ cancel out in this graph. For higher voltages, the normalized amplitudes get smaller, i.e., have a smaller-than-linear dependence on the applied voltage. Plotted in the inset in Fig. 5 are the amplitudes for the peak $(t=3.5 \mathrm{ps})$ and the slow component $(t=8$ ps). A good fit can be obtained for assuming a $V_{e o} \propto \sqrt{V_{0}}$ dependence. This demonstrates that the fast component is not due to an optical nonlinearity in the semiconductor, namely a 


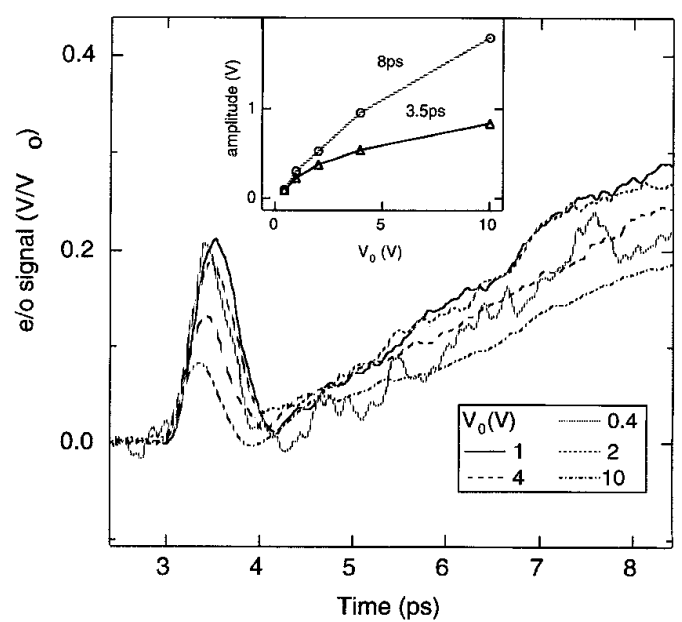

Fig. 5. E/o sampling data for different voltages on SI GaAs substrate. Pump wavelength is $760 \mathrm{~nm}$ and $P_{o p t}=20 \mathrm{~mW}$. Signals are normalized to the applied voltage. The inset shows the amplitude dependence for the fast (at $3.5 \mathrm{ps}$ ) and slow (at $8 \mathrm{ps}$ ) component.

$\chi^{(2)}$ or $\chi^{(3)}$ nonlinearity. In this case, the amplitudes would depend at least linearly on the applied electric field.

In contrast to the pump power dependence, the data in Fig. 5 show a qualitative difference versus $V_{0}$. For increasing $V_{0}$, the ratio of fast-to-slow components gets smaller and the duration gets faster ( $522 \mathrm{fs}$ for $1 V_{0}, 445 \mathrm{fs}$ for $10 V_{\mathrm{ac}}$ ).

This effect of pulse shortening and decreasing amplitude can be due to more carriers being accelerated and then scattered into the satellite valleys. The lower mobility in the satellite valley would effectively prevent the scattered carriers from any further contribution to the signal during this initial $\sim 1$ ps interval. Alternatively, as the energy stored in the PCS increases quadratically with the voltage $\left[E=(1 / 2) C V^{2}\right]$, the PCS is relatively less discharged by the accelerated carriers for higher voltages. More energy stored in the PCS could result in larger carrier acceleration. We will discuss this aspect in further detail in Section VII. Next, we investigate the influence of the band structure in the following chapter.

\section{WaVElEngth DEPENDENCE}

One possible explanation for the observed transients in $\mathrm{GaAs}$ is the scattering of the electrons into satellite valleys with lower mobility $(\sim 10 \times)$. The lower mobility would then result in a significant decrease of the conductivity. The scattering time constant has been measured to be 33 fs, i.e., instantaneous given our time resolution [14]. To investigate the influence of the band structure, we measured the electrooptic response for different pump wavelengths (Fig. 6). For a wavelength of $\lambda=620 \mathrm{~nm}(2 \mathrm{eV})$, the energy of the photoexcited charge carriers is sufficient to scatter into the $L$ and $X$ satellite valleys, even in the absence of an accelerating voltage. Whereas for $760 \mathrm{~nm}$, a direct transfer (without gaining additional energy from the applied field) is not possible. Within the limits of reproducibility for measurements with two different laser systems, the two measurements are identical.

At $\lambda=760 \mathrm{~nm}$, electrons are generated with an energy of $100 \mathrm{meV}$ below the $L$ valley. If we assume ballistic

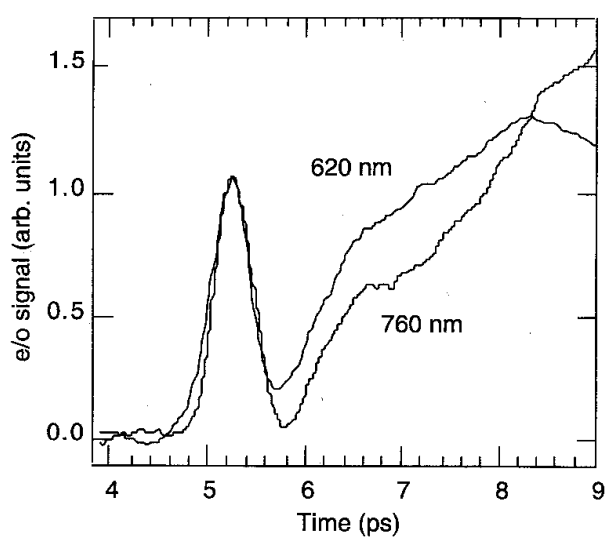

Fig. 6. Comparison of e/o data for different excitation wavelengths, $\lambda=$ $620 \mathrm{~nm}(\mathrm{CPM})$ and $760 \mathrm{~nm}$ (Ti : sapphire).

acceleration, the time, $t$, needed to acquire this energy is

$$
t=\frac{\sqrt{2 m^{*} E}}{e \varepsilon}
$$

where $m^{*}$ is the effective electron mass, $E$ the kinetic energy, and $\varepsilon$ the average field (here $\varepsilon=10 \mathrm{kV} / \mathrm{cm}$ ). With these values we obtain $t=280 \mathrm{fs}$, which should result in an observable delay in our measurement. However, at the electrodes, the electric field and hence the acceleration can be significantly enhanced [15]. An influence of the band structure cannot be completely ruled out for GaAs from this measurement. But given that a similar signal was previously observed on undamaged SOS, this result is further evidence that the band structure is not important for pulse generation.

\section{Position and Polarity Dependence}

\section{A. Pump Beam Position}

Fig. 7 shows the effect of polarity for a SI GaAs sample with the sign indicating the polarity of the adjacent electrode. Although changing the polarity contains information similar to changing the position of the pump beam, there is a subtle difference. Changing the polarity changes the "position" of the pump and the probe beam together. The probe beam position dependence will be discussed later.

Again, the pump and probe beams are close to the indicated electrode. The difference between the positive and the negative electrode has been attributed to the influence of Schottky barriers [2]. For an assumed p-type autodoping, the higher field is indeed expected at the positive electrode. However, for the high fields applied here $(10-20 \mathrm{kV} / \mathrm{cm})$, we do not expect to see a significant contribution from the Schottky barrier field, i.e., band bending should be negligible on SI material. The small influence of the contact field will be investigated later by measurements on an $n$-doped sample.

First, a closer look at the pump-beam position dependence is shown in Fig. 8(a). Here, the dependence on the pump beam ( $\sim 3-\mu \mathrm{m}$ diameter) position for a 10- $\mu \mathrm{m}$ gap transmission line with the probe beam near the positive electrode. Fig. 8(b) shows the geometry; the tick marks between the transmission 


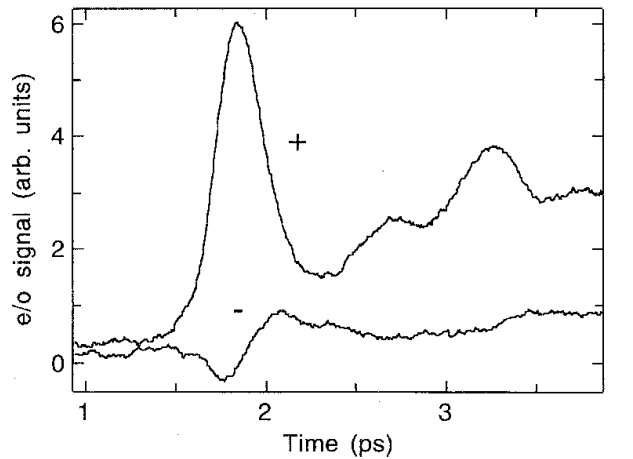

Fig. 7. Dependence of e/o-signal on polarity for an electrode spacing of 5 $\mu \mathrm{m}$ on SI GaAs substrate. Pump and probe beam positions are at the indicated electrodes.

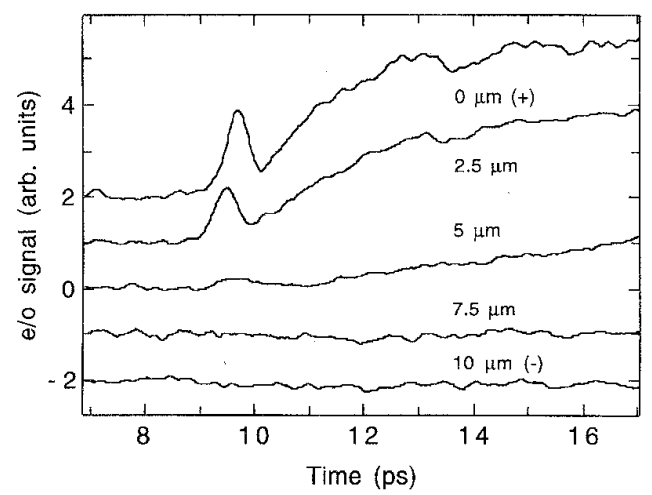

(a)

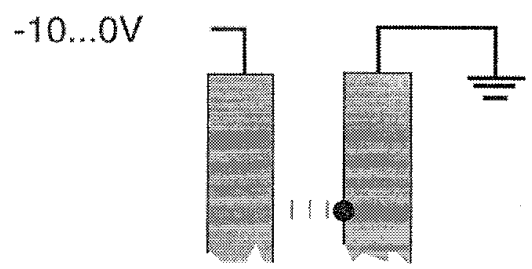

$10 . .0 \mu \mathrm{m}$

(b)

Fig. 8. (a) Dependence on the pump beam position. The electrode spacing is $10 \mu \mathrm{m}$ and the pump beam position is changed from the positive electrode $(0 \mu \mathrm{m})$ to the negative electrode $(10 \mu \mathrm{m})$. An arbitrary offset is added to the curves. (b) Geometry for the measurement in (a).

lines indicate the pump beam positions. The results can be summarized as follows: a short pulse is only measured when the pump beam is at least slightly overlapping the positive electrode. The signal disappears completely at the negative electrode as opposed to the measurements of Fig. 7, for spacing $s=5 \mu \mathrm{m}$. This result indicates that the nonuniform distribution of the static field does indeed have an influence for $s=10 \mu \mathrm{m}$.

\section{B. Probe Beam Position}

In order to have a well-defined probe position, the setup was modified for this measurement. The probe beam is not reflected

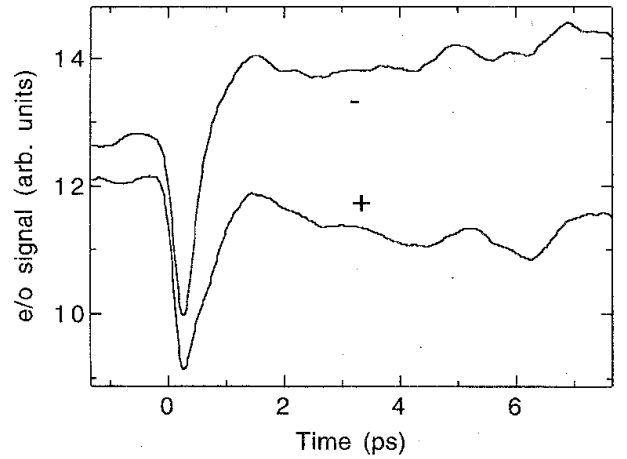

Fig. 9. Dependence on probe position. Pump beam is slightly overlapping the positive electrode. Substrate material is InP, electrode spacing is $10 \mu \mathrm{m}$, and pump beam diameter is $3 \mu \mathrm{m}$. The offset due to the modulated bias is not subtracted and the sign is not reversed.

by total internal reflection, because in this case, scattered laser light from the bottom facet can only be seen if there are imperfections in the e/o crystal. In this measurement, the probe beam is perpendicularly reflected from the gold electrodes and positioned at the inner edge of the electrode. Fig. 9 shows the dependence on the probe beam position for excitation at the positive electrode. In the first $500 \mathrm{fs}$, we observe a negative pulse at both probe positions. For longer times, the signal (i.e., the field) increases at the negative electrode and decreases at the positive electrode where the pump beam is positioned. The waveform in Fig. 9 was observed on InP. Qualitatively, the data show no difference compared to GaAs measurements. We did not, however, investigate samples with the exact same preparation that would allow quantitative comparison. We believe that the following interpretation of this result is crucial for understanding the short-pulse generation.

In the common transmission line model [9], a coplanar strip line of equal width and spacing on GaAs substrate has a capacitance of approximately $100 \mathrm{pF} / \mu \mathrm{m}$ (depending in detail on the frequency and the thickness of the electrodes). For an illuminated length of $2 \mu \mathrm{m}$, this results in a capacitance of 0.2 $\mathrm{fF}$. For $V_{0}=10 \mathrm{~V}$, the stored energy $\left[E_{c}=(1 / 2) C V^{2}\right]$ is 10 $\mathrm{fJ}$ and the charge $(Q=C V)$ equivalent to 12500 electrons. We follow the arguments of Sha et al. [16] for discussing the energy balance in a PCS, except we consider only the energy that is converted into kinetic energy of the carriers and neglect the energy radiated from the accelerated carriers. We consider the holes as immobile on this time scale. As in Section V, we assume ballistic acceleration and estimate the time that $N$ carriers need to acquire the energy stored in the capacitor, as

$$
t=\frac{\frac{\sqrt{2 m^{*} E_{c}}}{N}}{e \varepsilon} .
$$

The lowest excitation power that we used, $2 \mathrm{~mW}$, corresponds to $10^{8}$ photons/pulse. The absorption at the excitation wavelength is about $2 \mu \mathrm{m}^{-1}$. Therefore, we assume that all photons generate electron hole pairs in the field region of the PCS and set $N=10^{8}$. (This is the only instance where we overestimate the energy. We also assume here that the generation mechanism is instantaneous.) In this case, $E_{c}$ is 


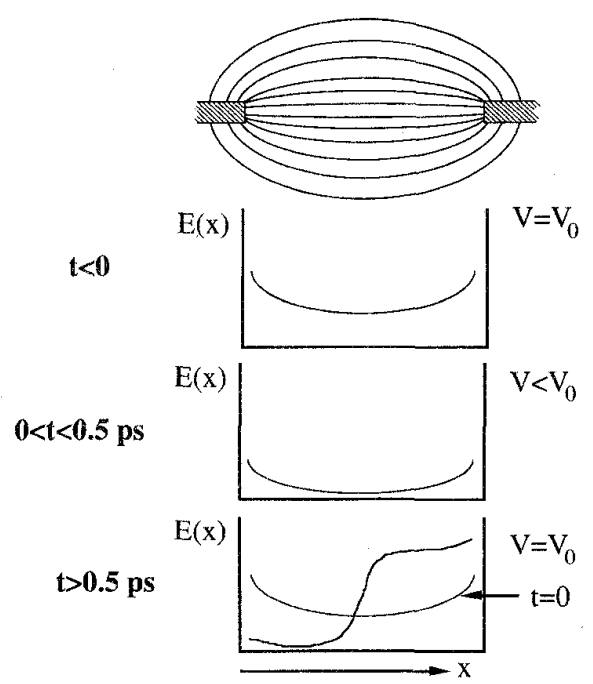

Fig. 10. Visualization of the three phases measured in Fig. $9 . t<0$ : without photo induced carriers the voltage is $V_{0}$. The field distribution is sketched with field enhancement at the electrodes but without asymmetry. $0<t<0.5$ ps: voltage breakdown. $t>0.5$ ps: voltage restored with local field screening on the left side where the carriers were generated. At the top of the graph, the position of the electrodes is shown with a sketch of the field lines. $E(x)$ denotes the amplitude of the total field depending on the position, $x$, between the contacts.

completely converted into kinetic energy of the accelerated carriers in $20 \mathrm{fs}$ ( $3.5 \mathrm{fs}$ for $P_{\text {opt }}=80 \mathrm{~mW}$ ). For recharging the PCS, we assume that the RC time constants are negligible and that recharging is only determined by the propagation speed on the transmission line, i.e., the negative voltage drop propagates away from the excitation area. The propagation velocity is 0.4 $\mathrm{c}$, and hence the voltage drop propagates across the diameter of the excitation spot of $2 \mu \mathrm{m}$ in less than $20 \mathrm{fs}$. After the PCS is recharged and $V_{0}$ is restored, local field screening by the separated carrier clouds determines the signal. These phases are sketched in Fig. 10.

\section{DOPED SubSTRATES}

Photoconductive switches on doped substrates are used to investigate the possible influence of Schottky barriers. A p-doped and an $\mathrm{n}$-doped sample with nominal doping levels of $5 \times 10^{15} \mathrm{~cm}^{-3}$ were compared. Again, the e/o signal for both samples are strongest at the positive electrode. With the photoconductive switch viewed as two back-to-back Schottky diodes in series, this p-doped metal-semiconductormetal structure has the higher field at the positive electrode and the n-doped material at the negative electrode. The Schottky depletion layer for zero bias is expected to be around $0.5 \mu \mathrm{m}$. So even for these low doping levels, we expect a pronounced static field asymmetry at least for low-bias voltages. From the data, however, we see that this does not determine the behavior in the first picosecond. For the case of an n-doped substrate, the pulse amplitude would be expected to be higher at the negative electrode if the amplitude was determined by the static electric field distribution. An interesting question is whether even in the $n$-doped case a higher pulse amplitude can be generated at the positive electrode. Therefore, we

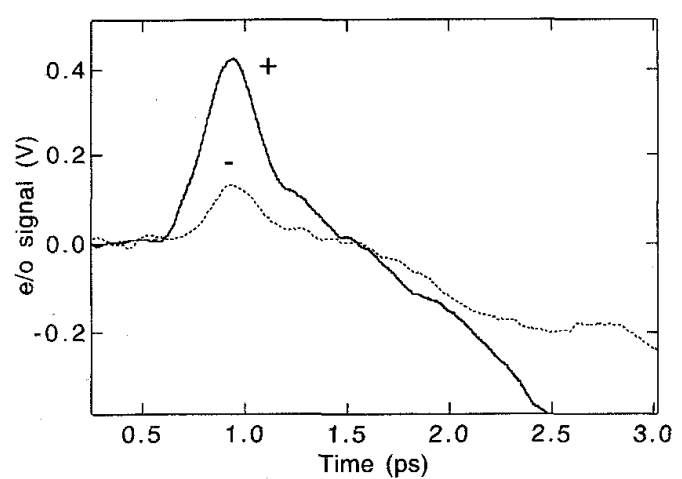

Fig. 11. Polarity dependence for $5 \times 10^{15} \mathrm{~cm}^{-3}$ n-doped GaAs. The electrode spacing is $10 \mu \mathrm{m}$. The wavelength of pump and probe beams is $770 \mathrm{~nm}$.

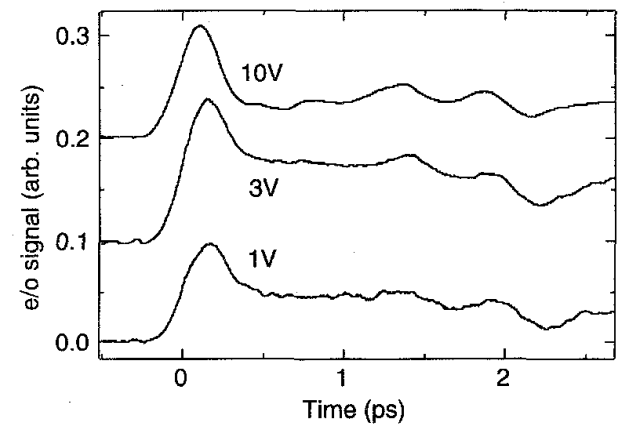

Fig. 12. E/o sampling data for a different bias voltage on a $5 \times 10^{15} \mathrm{~cm}^{-3}$ $\mathrm{n}$-doped substrate. An arbitrary offset is added to the curves.

present only measurements on an n-doped substrate. Fig. 11 shows that the generated pulse is again stronger at the positive electrode. This demonstrates that the observed asymmetry cannot be due to a static field nonuniformity. The probe beam position in Fig. 11 is closer to the negative electrode so the sign for longer time delay changes as for the top trace in Fig. 9.

Fig. 12 shows that the qualitative signal shape remains unchanged for different applied voltages. The influence of the Schottky depletion field is seen as a change of the offset in this measurement and shown in Fig. 13. The data are divided by the amplitude of the applied modulated voltage, so $V_{e o} / V_{0}=1$ denotes complete modulation. We assume that for the high voltages the voltage drop at the contacts is negligible. As only the field near the positive electrode is probed here, $V_{e o} / V_{0}=1$ also means that the local field is equal to the average field, indicating a uniform field distribution. This uniformity only applies to the lateral component (in the surface plane between the electrodes), as this is the component measured by the e/o sampling crystal. Fig. 13 demonstrates that the relative amplitude of the pulse is not directly correlated to the relative offset, which is a measure of the static field change at the probe position. For small amplitudes, the field nonuniformity does not influence the pulse shape and the pulse amplitude drops less than the offset. For high voltages, the field is fully modulated whereas the relative pulse amplitude decreases. Again, this shows that a static lateral field nonuniformity does not determine the pulse generation nonuniformity. 


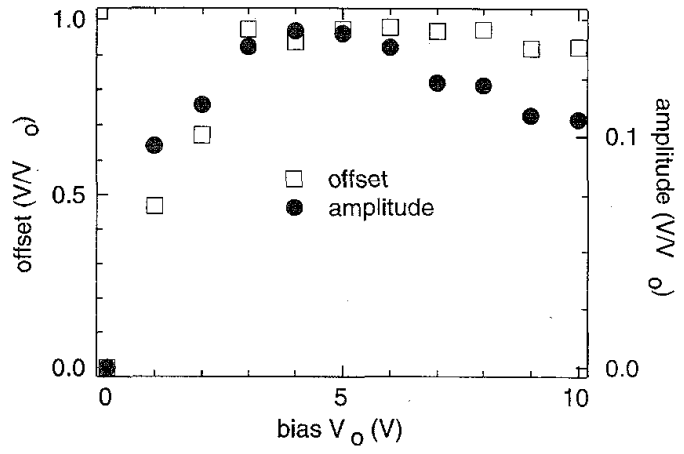

Fig. 13. Offset and amplitude of the fast component (at $200 \mathrm{fs}$ ) of the e/o signal in Fig. 12. An offset of $V / V_{0}=1$ indicates that the field is modulated between 0 and the average field. For small voltages, the voltage drop at the contacts reduces the signal.

Before we turn to another characterization method, it should be noted that it is extremely difficult to compare measurements on different samples quantitatively. As we demonstrated, the signal shape depends sensitively on the pump and the probe beam position. Furthermore, the probe beam position in the TIR geometry is not visible. We have therefore restricted our discussion to qualitative comparisons between different substrate materials and have discussed the dependencies on beam positions for different samples separately.

\section{BIAS-MODULATED REFLECTIVITY}

In order to rule out any effects that can be attributed to the sampling crystal and to achieve a smaller spatial resolution, we measured voltage-dependent reflectivity changes of the substrate, $\Delta R\left(V_{0}\right)$. We show the dependence of the position, $d$, between the electrodes, $\Delta R\left(V_{0}, d\right)$. The complication in using this method is that in addition to field changes, changes in the carrier density and in the energy of the charge carriers and contributions from optical nonlinearities are all measured at the same time. A detailed discussion of the setup and the interpretation of the data can be found in [17] and [18].

Fig. 14 shows the comparison of reflectivity changes for $\mathrm{CW}$ and for mode-locked laser light on a SI GaAs substrate. For the $C W$ light, we observe a symmetric, uniform $\Delta R$, whereas for the mode-locked light there is a strong peak at the positive electrode. In both cases, the wavelength and the average power are the same. The photon energy is above bandgap; the laser beam acts not only as a probe but also as a pump beam. The mode-locked measurement has an inherent time resolution. Reflectivity changes generated by the laser pulse due to the injection of carriers are only measured during the laser pulse of about $100 \mathrm{fs}$. The measurement compares the reflectivity with and without applied voltage. If surface and Schottky depletion fields are negligible compared to the applied field, then the signal can be interpreted as follows: we measure how effectively the carriers are swept out of the excitation (i.e., the observation) region with an applied voltage during the laser pulse duration. This interpretation accounts for the difference between the mode-locked and the $\mathrm{CW}$ measurement. As in the e/o measurements, we do not observe

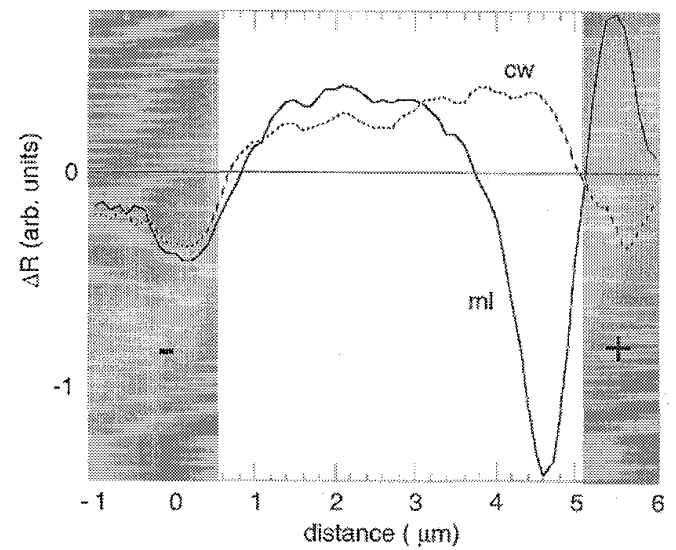

Fig. 14. Comparison of bias-modulated reflectivity of continuous wave (CW) and mode-locked (ml) light. The photon energy is above the band gap.

any static nonuniformity. Only ultrafast carrier acceleration leads to an enhancement of the signal at the positive electrode.

We have also measured reflectivity changes in the same geometry on an $\mathrm{Al}_{0.2} \mathrm{Ga}_{0.8}$ As substrate [18]. In this case, the photon energy of the probe light was below the band gap to rule out the influence of photogenerated carriers. For moderate light intensities, we did not measure any asymmetry. At high intensities, we observed second harmonic generation at the positive electrode and measured waveforms consistent with above-gap excitation.

\section{CONCLUSION}

The short pulses observed in asymmetric excitation are caused by a rapid voltage breakdown across the PCS. It is necessary to realize that the voltage across the PCS is not constant during the acceleration of the carriers. The kinetic and radiation energy can only be provided by the electric field. As the amount of stored energy is small compared to these energies, the voltage must drop. To understand the asymmetric response of PCS, it is crucial to take into account the 2-D nature of the coplanar setup. The field enhancement at the electrodes together with the different mobilities of electrons and holes account for the asymmetric behavior of PCS on a $5-\mu \mathrm{m}$ scale. On this length scale, we measure a pulse generation asymmetry without a static nonuniform lateral-field distribution. For larger devices where space charge regions are comparable to or smaller than the gap size, the static asymmetry can have an influence on the dynamic asymmetry. In this sense, our conclusions do not directly contradict an explanation that assumes a trap enhanced field for GaAs, or a model that artificially introduces a space charge layer at the electrodes. However, from the observation of dynamic processes like $\mathrm{THz}$ radiation or a transient e/o signal alone one can not deduce static properties like the field distribution.

The ultrafast pulses together with the enhancement at the positive electrode for $5-\mu \mathrm{m}$ gaps have been observed on substrate materials SI GaAs, LT GaAs, n- and p-doped GaAs, and InP. The pulse generation mechanism is independent of material properties, i.e., deep traps or dopants, that may influence the static field distribution. 


\section{REFERENCES}

[1] S. Gupta, J. Pamulapati, J. Chwalek, P. K. Bhattacharya, and G. Mourou, "Subpicosecond photoconductivity in III-V compound semiconductors," in Ultrafast Phenomena VII, Springer Ser. Chem. Phys. Berlin: Springer, 1990, vol. 53, pp. 297-299.

[2] D. Krökel, D. Grischkowsky, and M. B. Ketchen, "Subpicosecond electrical pulse generation using photoconductive switches with long carrier lifetimes," Appl. Phys. Lett., vol. 54, pp. 1046-1047, 1989.

[3] U. D. Keil and D. R. Dykaar, "Electro-optic sampling and carrier dynamics at zero propagation distance," Appl. Phys. Lett., vol. 61, pp. 1504-1506, 1992.

[4] S. Alexandrou, C.-C. Wang, R. Sobolewski, and T. Y. Hsiang, "Generation of subpicosecond electrical pulses by nonuniform illumination of GaAs transmission-line gaps," IEEE J. Quantum Electron., vol. 30, pp. $1332-1338,1994$.

[5] E. Sano and T. Shibata, "Mechanism of subpicosecond electrical pulse generation by asymmetric excitation," Appl. Phys. Lett., vol. 55, pp. 2748-2750, 1989.

[6] X. Zhou, S. Alexandrou, and T. Y. Hsiang, "Monte Carlo investigations of the intrinsic mechanism of subpicosecond pulse generation by nonuniform illumination," J. Appl. Phys., vol. 77, pp. 706-711, 1995.

[7] N. Katzenellenbogen and D. Grischkowsky, "Efficient generation of 380 fs pulses of $\mathrm{THz}$ radiation by ultrafast laser pulse excitation of a biased metal-semiconductor interface," Appl. Phys. Lett., vol. 58, pp. 222-224, 1991.

[8] S. E. Ralph and D. Grischkowsky, "Trap-enhanced electric fields in semi-insulators: The role of electrical and optical carrier injection," Appl. Phys. Lett., vol. 59, pp. 1972-1974, 1991.

[9] U. D. Keil, D. R. Dykaar, A. F. J. Levi, R. F. Kopf, L. N. Pfeiffer, S. B. Darack, and K. W. West, "High-speed coplanar transmission lines," IEEE J. Quantum Electron., vol. 28, pp. 2333-2342, 1992.

[10] D. R. Dykaar, T. Y. Hsiang, and G. A. Mourou, Picosecond Electronics and Optoelectronics, Springer Ser. in Electrophysics, G. A. Mourou, D. M. Bloom, and C. H. Lee, Eds. Berlin: Springer, 1985, 21, p. 249.

[11] M. B. Ketchen, D. Grischkowski, T. C. Chen, C.-C. Chi, I. N. Duling, III, N. J. Halas, and J. M. Halbout, "Generation of subpicosecond electrical pulses on coplanar transmission lines," Appl. Phys. Lett., vol. 48 , pp. 751-753, 1986.
[12] M. Y. Frankel, J. F. Whitaker, G. A. Mourou, and J. A. Valdmanis, "Experimental characterization of external electrooptic probes," IEEE Microwave Guided Waves Lett., vol. 1, pp. 60-62, 1991.

[13] P. C. M. Christianen, P. J. van Hall, H. J. Bluyssen, and J. H. Wolter, "Ultrafast carrier dynamics at a metal-semiconductor interface studied by femtosecond luminescence spectroscopy," Semicond. Sci. Tech., vol. 9, pp. 707-709, 1994.

[14] P. C. Becker, H. L. Fragnito, C. H. Brito Cruz, J. Shah, R. L. Fork, J. E. Cunningham, J. E. Henry, and C. V. Shank, "Femtosecond intervalley scattering in GaAs," Appl. Phys. Lett., vol. 53, pp. 2089-2091, 1988.

[15] E. H. Böttcher, F. Hieronymi, D. Kuhl, E. Dröge, and D. Bimberg, "Transient response of lateral photodetectors," Appl. Phys. Lett., vol. 62, pp. 2227-2229, 1993.

[16] W Sha, J-K Rhee, T. B Norris, and W. J. Schaff, "Transient carrier and field dynamics in quantum-well transport: From the ballistic to the quasiequilibrium regime," IEEE J. Quantum Electron., vol. 28, pp. 2445-2455, 1992.

[17] U. D. Keil, D. R. Dykaar, R. F. Kopf, and S. B. Darack, "Reflectivity measurements of femtosecond carrier and field dynamics in semiconductors," Appl. Phys. Lett., vol. 64, pp. 13-15, 1994.

[18] _ "Femtosecond reflectivity measurements and second harmonic generation in nonresonant excitation of photoconductive switches," Appl. Phys. Lett., vol. 64, pp. 1812-1814, 1994.

U. D. Keil, photograph and biography not available at the time of publication.

D. R. Dykaar (S'86-M'87-SM'93), photograph and biography not available at the time of publication. 